\title{
DIE ZUKUNFT DES WASSERS AUF UNSEREM PLANETEN ÜBERDENKEN
}

\section{REVIEW ARTICLE}

DIAS, Deusira Nunes Di Lauro ${ }^{1}$

DIAS, Adailton Di Lauro ${ }^{2}$

DIAS, Deusira Nunes Di Lauro. DIAS, Adailton Di Lauro. Die Zukunft des Wassers auf unserem Planeten überdenken. Revista Científica Multidisciplinar Núcleo do Conhecimento. 04-Jahr, Ed. 08, Vol. 06, S. 19-32. August 2019. ISSN: 2448-0959

\section{ZUSAMMENFASSUNG}

Wasser gilt als die wichtigste, kostbarste und unentbehrlichste Flüssigkeit für das Überleben des menschlichen Lebens auf der Erde und ist ein Grund zur ständigen Sorge, weil die Art und Weise, wie die Gesellschaft Wasserressourcen auf dem Planeten angeeignet und genutzt hat, Engagiert für Qualität und den Zugang vieler Gemeinschaften zu dieser lebenswichtigen Ressource für die Erhaltung des Lebens. Diese Arbeit zielt darauf ab, Strategien zu analysieren und zu diskutieren, die die Erhaltung und nachhaltige Nutzung von Wasser auf dem Planeten gewährleisten, wobei zu adressieren ist, dass in einigen Regionen Brasiliens und der Welt bereits extreme Wasserknappheit und, nach einigen Autoren, dieses Problem neigt dazu, mehr und mehr aufgrund mehrerer Faktoren zu eskalieren, und die wichtigsten sind

\footnotetext{
${ }^{1}$ Abschluss im Rahmen von Briefen der Staatlichen Universität Bahia - UNEB Campus X; Postgraduierte in Portugiesisch von der Universität Vale do Cricaré und ein regelmäßiger Student des Master-Abschlusses in Bildung von Universidade Internacional Unigrendal online.

${ }^{2}$ Graduierte in Briefen von Uneb, Graduierte in Englisch von Unimes, Postgraduierten an den Integrierten Colleges von Jacarepagua (FIG) und regelmäßige Student des Master-Abschlusses in Bildung an der International University Unigrendal online.
} 
das schnelle Wachstum der Bevölkerung und die große Nachfrage nach Nahrungsmitteln in der Welt. Die Wasserressourcengesetze werden zunehmend angepasst, um die Wasserressourcen und die gesamte Umwelt zu erhalten. Brasilien hat gezeigt, dass es notwendig ist, zu erhalten, wie auch immer noch viel zu tun bleibt, um die Auswirkungen des Menschen auf die Umwelt zu minimieren.

Schlagworte: Wasser, Knappheit, Konservierung, Nachhaltigkeit.

\section{EINFÜHRUNG}

Die Menschheit hat, vor allem in diesem Jahrhundert, unzählige Errungenschaften erreicht, die sich Wissenschaft und Technologie nie vorstellen konnten. Das Problem des Wassers und der Erhaltung des Lebens auf dem Planeten hat jedoch einen Großteil der Weltbevölkerung dazu gebracht, sich zu fragen, was zu tun ist, um sicherzustellen, dass jeder Zugang zu Wasser hat, aber es hat nicht immer eine Antwort, weil der Mensch nicht immer oder fast nie D sein soll. Bericht über die Notwendigkeit, dieses sehr kostbare Gut zu bewahren.

Diese Sorge in der Zukunft kommt nicht von ungefähr, schließlich zeigen Daten über die Verteilung, Reserve und Verfügbarkeit von Wasser auf dem Planeten die Notwendigkeit, Alternativen für die Erhaltung zu suchen, wie VICTORINO sagt:

Es ist bekannt, dass fast die gesamte Oberfläche des Planeten Erde von Wasser bedeckt ist: von den Ozeanen, Flüssen und Seen, Bächen und Sanghas. Wasser aus polaren Eiskappen, Regenwasser, viel, viel Wasser... Aber in Wirklichkeit ist nicht alles blau (die Farbe, die die Erde bedeckt - aufgrund des Wassers, wenn es aus dem Weltraum gesehen wird), weil das gesamte Wasser auf dem Planeten (1,37 Milliarden km 3) im Grunde aus zwei Arten besteht: Salzwasser der Meere und Süßwasser von Flüssen, Seen und Untergrund. $97 \%$ der Gesamtmenge sind jedoch mit Salzwasser belegt, das für den Verzehr ungeeignet ist. Mit dem sogenannten "Wasserkreislauf" (Prozess der Verdunstung und Rückführung von Wasser an die Oberfläche in Form von Regen, Schnee, 
Hagel oder Frost) setzt sich das Wasser auf verschiedene Weise neu zusammen, um Pflanzen leben zu lassen und mehrere Biomms nach dem natürlichen Verlauf des Lebens zu erzeugen. Die Natur ist klug und gerade weil, wenn Wasser in Form von Verdunstung in die Atmosphäre entweicht, das Schwitzen durch Niederschlag kompensiert wird. Dieser Unterschied zwischen dem Wasservolumen, das fält, und dem Wasservolumen, das verdunstet, beträgt etwa 45000 Kubikkilometer pro Jahr - was der Mensch theoretisch ausgeben könnte. Nur $20 \%$ dieser Summe sind verfügbar. Nutzbares Wasser ist in Flüssen, Seen, Regenwasser und unterirdischem Wasser. Sie machen jedoch zusammen nur $1 \%$ des Süßwasservolumens aus (VICTORINO, 2007, S. 16).

Abbildung 1-Diagramm-"Wasserverteilung auf der Erde"

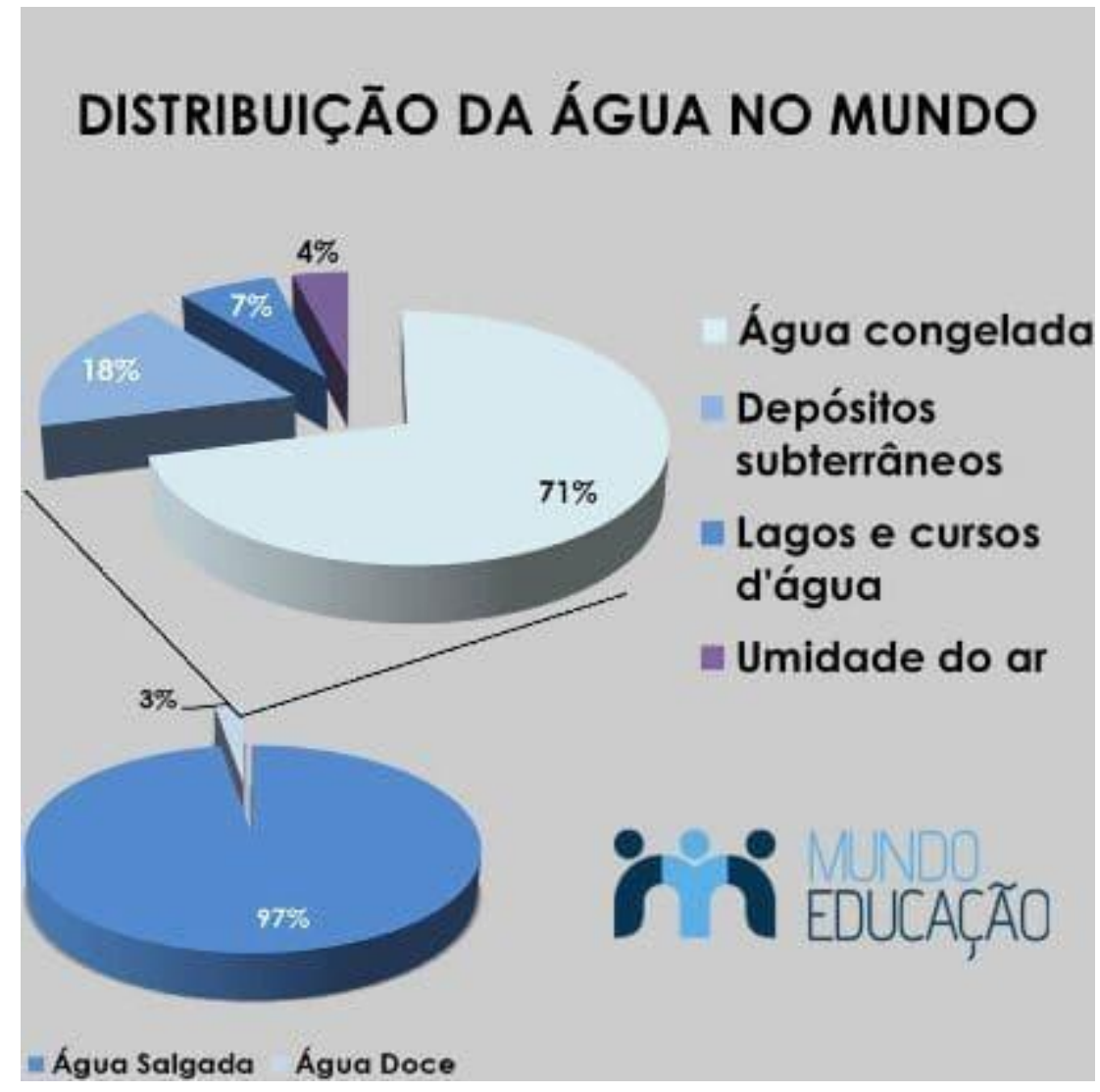

(Quelle: World Education Site) 
Bei der Analyse des obigen Diagramms wird beobachtet, dass die zur Verwendung verfügbare Wassermenge nicht ausreicht, da:

Wenn wir 1,5 Liter Wasser, wie wir es auf dem Planeten finden, sammeln und proportional teilen, würde die verfügbare Menge an Süßwasser einem einzigen unbedeutenden Rückgang entsprechen. Und um dies zu verkomplizieren, wird dieser kleine Rest zunehmend verschmutzt, vor allem in den großen Städten, aufgrund der Zunahme der Weltbevölkerung, die erst im letzten Jahrhundert dreimal größer wurde, was die Zunahme von Fabriken, mehr Abfall und mehr Bewässerung verursachte In den Kulturen. Nach Angaben der Weltbank haben heute etwa 80 Länder mit Versorgungsproblemen zu kämpfen. Die kritischste Situation ist in Asien, wo $60 \%$ der Bevölkerung leben und nur $32 \%$ des verfügbaren Süßwassers zur Verfügung stehen (VICTORINO, S. 17).

Bei der Analyse all dieser Daten ist es möglich, die Schwere der Auswirkungen zu bewerten, die durch das Handeln des Menschen auf die Umwelt und speziell auf das Wasser verursacht werden, das als lebenswichtige Ressource für das Leben auf der Erde gilt. Viele Länder haben bereits durch Verträge und Abkommen Haltungen zur Eindämmung und Erhaltung eingeführt, um derartige Auswirkungen zu minimieren. Ein beträchtlicher Teil der Weltbevölkerung ignoriert jedoch immer noch, dass die Zukunft bedroht ist und dass es einen Wandel geben wird, nicht nur der Mentalität, sondern auch der Einstellung zur Nutzung dieser Ressource, die in unverantwortlicher Weise genutzt wurde, um lange Geschichte.

Es ist bekannt, daß die Weltbevölkerung ungeordnet gewachsen ist, und dies erfordert auch eine erhebliche Zunahme der industriellen landwirtschaftlichen Produktion, um Nahrungsmittel für alle zu garantieren, was die Umwelt zunehmend stark belastet. Angesichts dessen ist es notwendig, Strategien zu finden, die die Auswirkungen zunichte machen, da für das Jahr 2020 eine Bevölkerung von mehr als acht Milliarden Menschen erwartet wird: $65 \%$ in Küstengebieten und $60 \%$ in Städten mit mehr als 2,5 Millionen Einwohnern. Darüber hinaus hat die Nutzung der Wasserversorgung in kurzer Zeit enorm zugenommen: Während die Renovierung den 
Wasserressourcenstand von 1900 bis 1995 nicht änderte, erhöhte sich die menge der eingesetzten Mittel auf das Sechsfache (zweimal so hoch wie die Bevölkerungszuwachsindex) und das Zweifache seit 1975. Von dem gesamten Wasserverbrauch auf dem Planeten absorbiert die Landwirtschaft einen globalen Durchschnitt von $70 \%$ der Wasserversorgung, und in den unterentwickelten Ländern ist der Anteil sogar noch höher, indem er einen Anstieg von 80 bis $90 \%$ verzeichnet, wobei durchschnittlich $20 \%$ für die Industrie und $10 \%$ für den inländischen Verbrauch und die Tros.

Bei der Analyse der Situation Brasiliens in diesem Zusammenhang wird noch deutlicher, dass Wasser nicht rational genutzt wurde, was die Versorgung und die Lebensqualität in vielen Regionen des Landes beeinträchtigt und sehr stark beeinträchtigt.

\section{WASSERVERBRAUCH IM LAND}

Abbildung 2-Diagramm - Wasserverbrauch im Land

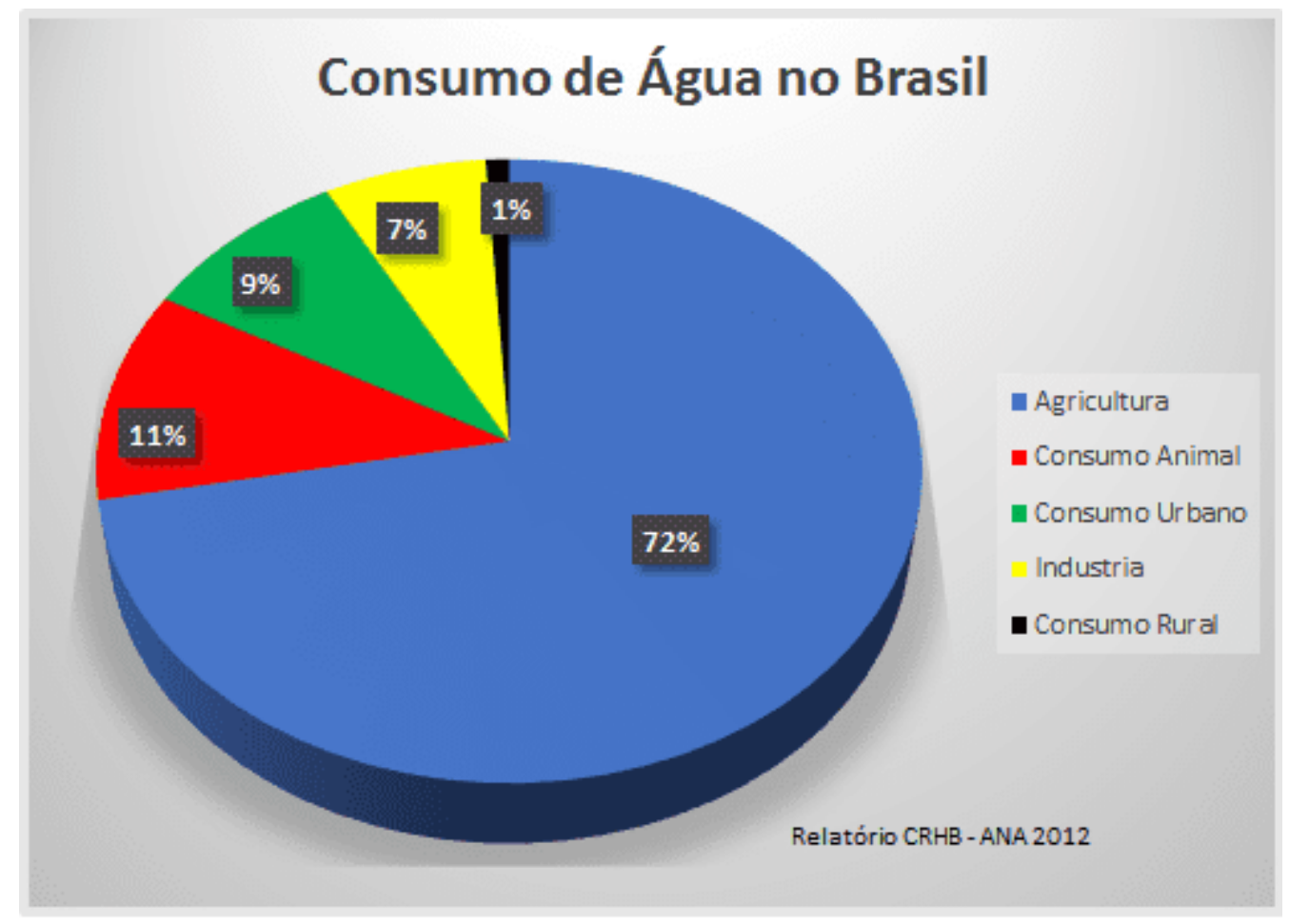

(Quelle: EOS Consultants) 
Die obige Grafik zeigt die Landwirtschaft als den großen Bösewicht beim Verbrauch und der Verschwendung von Wasser in Brasilien und obwohl das Land ein Pionier bei der Annahme von Gesetzen ist, die den Verbrauch begrenzen und einige Sanktionen gegen diejenigen verhängen, die Missbrauch beim Verbrauch und der Verwendung von Wasser begehen, ist es auch bekannt, dass es eine unzureichende Aufsicht seitens der Herrscher gibt, um sicherzustellen, dass die Gesetze eingehalten werden, das heißt, obwohl es Gesetze der Erhaltung gibt, ist in der Praxis noch lange nicht geschehen.

Trotz des rechtlichen Konzepts der guten gemeinsamen Nutzung der Menschen ist Wasser heute für viele eine Ressource von wirtschaftlichem Wert. Dies resultiert aus der steigenden Nachfrage nach Wasser für die verschiedenen Nutzungen, die die städtische und industrielle Entwicklung der Länder begleitet. Heutzutage treten die großen Probleme im Zusammenhang mit dem Wasser nicht aufgrund der Natur auf, sondern entstehen durch Missbrauch mit Abfällen und Unvorsorge sowie durch weit verbreitete Umweltverschmutzung.

Die Wasserordnung, das Gesetz größer als Disziplin die Verwendung von Wasser in Brasilien ist 10. Juli 1934, ist bereits ein bestimmtes Alter, aber nicht so ist es nicht mehr aktuell, weil es rechtliche Kategorien klassifiziert, diskriminiert die Verwendung von Wasser und seine Vorschriften und, vor allem: zielt darauf ab, die allgemeinen Interessen der Gesellschaft zu gewährleisten. Im alten Code war es in einigen Fällen möglich, eine private Wasserdomäne zu finden, aber diese wurde ab Oktober 1988 ausgehoben, als alle Körper D'Wasser öffentlich wurden.

\section{DIE QUALITÄT DES WASSERS, DAS WIR VERBRAUCHEN}

Die Qualität des auf dem Planeten verfügbaren Wassers ist ein weiterer Aspekt, der auch von den Gesundheitsbehörden weltweit besorgniserregend war, da Krankheiten bei der Verwendung von dekontaminiertem Wasser vermieden werden. Die Qualität des Wassers hat das Glück einiger Regionen auf der ganzen Welt in wirtschaftlichem Charakter entschieden, vor allem in Bezug auf die Herstellung von Bieren. Aber nicht nur in der Branche der Brauereien ist die Reinheit des Wassers wichtig, es ist auch in 
den Industrien der Wandteppiche, in der Gerberei, in der Gewebeindustrie und in den Edelsteinen notwendig. Darüber hinaus wird die Qualität des Wassers die Lebensqualität seiner Verbraucher bestimmen. Wenn es kein geeignetes Trinkwasser für den menschlichen Verzehr gibt, ist das Ergebnis die hohe Rate von Krankheiten und anderen Problemen, die sich daraus ergeben. Dies ist eine Sorge, die in der Vergangenheit den Erfolg oder Misserfolg der am weitesten entwickelten Nationen bestimmt hat, wie Célia Jurema sagt:

Der Mensch kümmerte sich immer um wasser. 4000 Jahre v. Chr. waren die ersten Gesetze, die bekannt waren, Codes, die die Verwendung von Wasser regelten, geschrieben von den Sumerern. Aber nicht alle Zivilisationen waren vorsichtig damit. Aufzeichnungen zeigen, dass die Maya die Stadt Tical verlassen musste, die sich in einem vollen tropischen Wald befand, wo die Ruinen der Sonnenpyramide gefunden wurden, weil sie nicht wussten, wie sie das Wasser richtig speichern sollten, abgesehen von wachsenden Erosionen und großen Entschmieden, weil sie Holz auch in den inneren Strukturen der Säulen ihrer gigantischen Tempel verwendet. In Mexiko, auf der Halbinsel Yucatan, wurde der Regengott Chac verehrt, und wenn das Wasser knapp wurde, wurden neue Tempel errichtet, in der Hoffnung, dass der Gott der Gewässer die Maya vor Dürren retten würde. Sie sind oft gefunden, Motive der Maya Regen Gott an der Basis der Pyramiden. Die Koexistenz mit den Flüssen brachte Weisheit in die Bevölkerung während der gesamten Entwicklung der Menschheit, die Pharaonen, plante ihre Städte verlassen die Flussgebiete Frei " (VICTORINO, 2007, S. 19).

Seit weiter entfernten Zeiten ist Wasser immer einer der wichtigsten sozialen Regulatoren. Die Strukturen der bäuerlichen Gesellschaften und Dorfgemeinschaften, in denen die Lebensbedingungen eng mit dem Boden verbunden sind, wurden um das Wasser herum organisiert. Es gab seltene Fälle, in denen alle Mitglieder einer Gemeinschaft in Bezug auf Wasser auf dem gleichen Niveau waren - der Zugang zu 
wassernahem Wasser war fast immer mit Ungleichheit verbunden. Dies führt uns zu verstehen, wie es die Beziehung der Menschen mit Wasser und zueinander durch Wasser ist. Der Mann brauchte jedoch eine lange Zeit, um sich um die Qualität des Wassers zu kümmern und sich effektiv um sie zu kümmern - viele wussten noch heute nicht, dass, mehr Tage weniger Tag, Knappheit Realität sein wird, und weiterhin Wasser indiskriminell zu verwenden ist für kleinere Aufgaben Es ist, wie man das Auto mit Schlauch waschen oder den Bürgersteig mit Jets D ' Wasser fegen. Andere spielen noch immer allerlei Abfall in Seen, Flüssen und Bächen. Wie lange wird die Gesellschaft noch so unbedeutend und unverantwortlich handeln? Das Wasser, dieses Mineral, das wir beide kennen und das teil von uns ist, existiert seit mindestens 3,9 Milliarden Jahren. Das "rationale Wesen" hat es in einigen Generationen bereits geschafft, Kompromisse einzugehen, nicht nur die Qualität, sondern auch die Quantität dieses natürlichen Frühlings. Man kann den Entwicklungsgrad eines Volkes anhand der Qualität der Wasserversorgung und der sanitären Einrichtungen, die der Bevölkerung angeboten werden, bewerten. Die Fähigkeit, das menschliche Leben und die Gesellschaft zu unterstützen, ist komplex, dynamisch und variiert je nachdem, wie der Mensch mit Umweltressourcen umgeht. Es ist seltsam, dass die weisen Priester der Antike die bestehenden Zusammenhänge zwischen Wasserversorgung und Waldschutz nicht wahrnahmen. Zu dieser Zeit war die Pflanzung nur das Privileg der Fürsten, es war nicht durch ihre "glänzenden" Köpfe, die Fronten von Pflanzgefäßen erfordern würden, um die geschwärmten Länder wieder aufzuforse:

Vor 8000 Jahren hatte Brasilien 9,8 \% der Weltweiten Wälder. Zu Beginn des 21. Jahrhunderts sank dieser Prozentsatz auf $28 \%$, und schlimmer noch, die 64 Millionen Quadratkilometer der bestehenden Wälder vor der demografischen und technologischen Expansion der Menschen bleiben unter 15,5 Millionen, etwa 24 \%. Das bedeutet, dass mehr als 75 \% der Wälder der Welt bereits verschwunden sind... Ebc

Die Wiederherstellung degradierter natürlicher Ressourcen ist schwieriger als seine Erhaltung; Und viele von ihnen, wie Wasser, können nicht wiederhergestellt werden, wenn es endet, gibt es kein Zurück mehr, wenn es endet, ist für immer. Die falsche 
Illusion, dass Wasser niemals in der Natur enden wird, rechtfertigt vielleicht zum Teil die historische Vernachlässigung der Menschen in ihren Beziehungen zu den Wasserressourcen. Es ist bekannt, dass nicht so viel Trinkwasser zur Verfügung steht, wie die Landschaft uns sehen lässt. Was wir als Trinkwasser haben, sind nur 0,03 \% des gesamten Wassers auf dem Planeten. Diese unbedeutende Menge sollte alle erdenkliche Pflege erhalten, aber das ist nicht das, was wir auf fast jedem Kontinent sehen, schließlich werden die wichtigsten Grundwasserleiter mit einer schnelleren Geschwindigkeit als ihre natürliche Fähigkeit, sich zu erholen, erschöpft.

\section{DIE WAHLLOSE NUTZUNG VON WASSER UND DIE VERPFLICHTUNG DER LEBENSQUALITÄT DER MENSCHHEIT}

Es ist nicht notwendig, zu weit zu gehen, um Situationen der Verschwendung und der Abwertung der natürlichen Ressourcen wahrzunehmen. Mit dem Wasser ist immer noch das gleiche, und in der Theorie die Idee, diese Ressource zu erhalten, obwohl etwas betont, gibt es in der Praxis keine große Mobilisierung, um absehbare Folgen einer Zukunft mit Wasserknappheit zu verhindern. Das folgende Zitat definiert die betreffende Situation klar:

Eine der größten Herausforderungen der Gesellschaft in diesem 21. Jahrhundert ist die dringende Notwendigkeit der Erhaltung der Wasserressourcen in Qualität und Quantität, in einem Szenario des Bevölkerungszuwachses und der geringen Nachhaltigkeit der Produktionsprozesse und des Verbrauchs.

[...]Diese Prognosen des Bevölkerungswachstums und Schätzungen im Zusammenhang mit der Nahrungsmittelproduktion, -erhaltung und verteilung gehen davon aus, dass wir 70 \% der Einwohner des Planeten haben werden, wenn die Weltbevölkerung in den nächsten 50 Jahren 10 Milliarden Einwohner erreicht. Beseitigung von Mängeln in der Wasserversorgung, von denen etwa 1,06 Milliarden Menschen betroffen sind, die nicht einmal Wasser für Grundnahrungsmittel haben werden (SILVA, 2009, S. 1). 
Während in vielen Ländern der Welt das Wasser bereits zu einem Konflikt geworden ist, der zu diversen Konflikten geführt hat, hat Brasilien die große Verantwortung, die wir mit der Lebensqualität zukünftiger Generationen haben, noch nicht erkannt. Laut Célia Jurema Aito ist Brasilien ein privilegiertes Land in Bezug auf die Wasserverfügbarkeit, da es mit $28 \%$ der südamerikanischen Verfügbarkeit und $12 \%$ der weltweiten Wasserreserven zählt. Auf brasilianischem Gebiet befinden sich $72 \%$ des Wassers im Amazonasbecken. Der Amazonas hat eine Ausdehnung von 6.885 Kilometern und ist mit 175 Millionen Litern pro Sekunde im Atlantik der größte Wasservolumender der Welt. Es darf jedoch nicht vergessen werden, dass das Bevölkerungswachstum das Risiko von Knappheit auch bei uns bewirkt. Laut Célia Jurema stieg Brasilien zwischen 1970 und 2000 von einer städtischen Bevölkerung von $55 \%$ auf $82 \%$ der Gesamtbevölkerung. Es ist bekannt, dass mehr als 1,4 Milliarden Menschen keinen Zugang zu Trinkwasser haben und weitere 2 Milliarden keine grundlegende sanitäre Versorgung haben.

Als sich der moderne Mensch zur Technologie entwickelte, hörte er auf, Kontakt mit seiner natürlichen Essenz zu haben, lebte immer weiter von seiner Basis, der Natur, und wurde ein ängstliches und gereiztes Wesen. Das kollektive Ungleichgewicht, mit dem unser Planet konfrontiert ist, stellt ein Ungleichgewicht der Individuen dar, die die Weltbevölkerung ausmachen. Dieses Ungleichgewicht bezieht sich auf ein Versagen im Evolutionsprozess des Menschen, der sich von seinen Ursprüngen distanziert, die meiste Zeit sein individuelles Wohlergehen anstrebt und dabei vergisst, dass wir Teil eines lebendigen Organismus sind, der "Gesellschaft" genannt wird, und dass er Entwickeln Sie effektiv, ist es notwendig, das Wohl aller und nicht nur von sich selbst zu gewährleisten. In Großstädten wird wahrgenommen, dass sich immer mehr Menschen allein fühlen, hilflos, sogar mitten in der Menge leben, was sie jeden Tag individualistischer und egoistischer macht. Und obwohl es heutzutage eine Idee der Erhaltung gibt, zumindest in den Anzeigen vieler Unternehmen, die die Flagge der Erhaltung eines "Business Marketing" anheben wollen, das profitabel ist, ist die große Wahrheit, dass wenig für den Erhalt der Umwelt und Folglich Wasser im Land. Daher spüren wir die Ineffizienz unserer Herrscher, der Nichtregierungsorganisationen und sogar der Bevölkerung selbst in Bezug auf die Umweltfrage, trotz so vieler 
Bemühungen, da die ganze Arbeit im Wesentlichen zur Lösung der Probleme geleistet wird. Wenn der Prozess der Veränderung und Verbesserung sollte auf der internen Ebene jeder Person beginnen.

Leider ist die Weltbevölkerung, die immer mehr Platz auf dem Planeten einnimmt, direkt für die Verschlechterung von Flüssen, Küstengebieten, Ozeanen, Stränden verantwortlich, und da diese Bevölkerung nicht wächst, ist das Müllaufkommen von Tag zu Tag größer geworden. Um sich ein Bild von dem Problem zu machen, produzieren nur die Amerikaner 212 Millionen Tonnen Müll pro Jahr und bewegen fast 3 Millionen Plastikflaschen pro Stunde. Es braucht ein Gewissen, um diese Art von Müll an Orten zum Recycling zu entsorgen. In Brasilien legt die nationale Richtlinie über feste Abfälle (Bundesgesetz 12.305/2010) fest, dass alle Materialien, die von inländischen und kommerziellen Tätigkeiten erzeugt werden, die von öffentlichen Reinigungsdiensten gesammelt werden können, an den Bestimmungsort weitergeleitet werden müssen. Beenden Sie nur, wenn es nicht möglich ist, es wiederzuverwenden, entweder durch Recycling, Wiederverwendung, Kompostierung oder Stromerzeugung. Wo es keine praktikable Technologie gibt, müssen Abfälle für Deponien bestimmt werden. Von dem im Jahr 2016 produzierten Volumen verfügten jedoch fast 30 Millionen Tonnen nicht über die geeignete Bestimmung, was einem Prozentsatz von 41,6\% der insgesamt erzeugten Menge entspricht, was den festen Abfall in ein ernstes Umweltproblem verwandelt, da eine unzureichende Entsorgung Das Verladen dieses Materials in Bäche und Flüsse und erreicht folglich die Ozeane, zusätzlich zum Problem der Deponien, die Auswirkungen auf die öffentliche Gesundheit unter anderem negative Auswirkungen.

Im Durchschnitt werden 80 Millionen Tonnen Siedlungsabfälle produziert, davon fast 470 Kilo pro Einwohner, wobei $60 \%$ dieser Summe recycelbares Material sind. Allerdings werden nur $3 \%$ des produzierten Mülls für das Recycling weitergeleitet.

Der Eindruck ist, dass überall die Oberflächengewässer mit einer erschreckenden Vielfalt an städtischen, industriellen und landwirtschaftlichen Abfällen verschmutzt werden. Selbst in den Industrieländern, in denen Rechtsvorschriften über die Wasserqualität gelten, ist die Verschmutzung nach wie vor ein Problem, das sich um 
sie kümmert. In den Vereinigten Staaten wurde festgestellt, dass $60 \%$ der untersuchten Seen durch Nährstoffe aus Düngemitteln und Tierischem Geltendem verschmutzt sind, was neben der Verschlechterung der Wasserqualität auch fast massiven Fischsterben verursacht. In Kanada sind 20000 Seen von sauren Regenfällen betroffen.

Brasiliens demografisches und wirtschaftliches Wachstum in den letzten Jahren hat die Wasserressourcen weit über seine Kapazität hinaus genutzt, um sowohl Quantität als auch Qualität zu unterstützen. Die ernsten Probleme bei der Wasserbewirtschaftung werden durch die ungeordnete Bevölkerungszunahme in den Städten und die begrenzte Verfügbarkeit von Wasserressourcen verursacht, und darüber hinaus reichen die öffentlichen Maßnahmen zur Erhaltung der Gewässer angesichts der Verbrauchs- und Auslastungsbedarf. Die Besetzung der flachen Räume, in der Nähe der Flüsse und ohne Kontrolle, verursachte seit Jahren kleine Überschwemmungen in einer großen Anzahl von Städten im Süden und Südosten, in Brasilien. In den Jahren 80 kam es zu mehreren Überschwemmungen am Flussufer und größeren Schäden, vor allem in Parané, Santa Catarina, Sao Paulo und Minas Gerais. In unserem Land gibt es eine große Vielfalt von Situationen, mit vielen Wasserressourcen in den Regionen Nord und Mittlerer Westen und Knappheit im Nordosten und Südosten, im Gegensatz zu einer größeren Konzentration der Nachfrage, zusammen mit der südlichen Region. Die Stadtentwicklung erhöht auch wasserabweisende und absorptionsarme Bereiche durch Wohnhäuser, Spaziergänge, Straßen, Parkplätze und sogar Parks. Da Regenwasser nicht in den Boden eindringen kann, fließt das zusätzliche Volumen in das Entwässerungssystem in Richtung der Flüsse. Im Jahrzehnt der 70 war die Hoffnung groß, dass es möglich war, das Wachstum der Städte zu planen und ihre Zukunft harmonisch zu planen. 37 Jahre später scheint es jedoch, dass die Stadtplanung in Brasilien oder sogar im Ausland verkümmert war, was zur Anhäufung von Problemen führte.

Die brasilianische Uferpromenade ist das Opfer von Immobilienwut, vor allem in Rio de Janeiro, zusätzlich zu den Stränden des Nordostens und Südens. Rampant Wachstum bietet die Entstehung von Dörfern und Favelas, wo Trinkwasser nicht 
gefunden wird und, viel weniger, Abwasser. Rio de Janeiro und Sao Paulo, die beiden größten brasilianischen Städte, haben gravierende Defizite im Abwasserbehandlungsnetz und trotz internationaler Kredite die hohe Verschmutzung der Guanabara-Bucht und des Flusses Tieté nicht verringern. Aufgrund des mangelnden Bewusstseins der Bevölkerung und eines Umweltbildungsprogramms, das Veränderungen im Verhalten von Individuen anregt.

Die größten Städte des Landes verwalten ihre Wasserressourcen schlecht. 1997 bot die Weltbank 40 Milliarden US-Dollar für Sanitärunternehmen an. Fast 100\% des Geldes bleiben noch intakt, weil brasilianische Unternehmen nicht unter die technischen Mindestanforderungen fallen, um Die Kredit-Bestätigung des Geologen Aldo Rebouas von der Mineral Resources Research Company zu erhalten - dies, weil die Bank Bestimmt, dass Unternehmen einen Verlust von maximal $20 \%$ an Wasserverteilungsnetzen haben. Allein in Sao Paulo betrugen die Verluste im Jahr $200130 \%$.

Die Umweltzerstörung ist einer der kritischsten Aspekte des Verschlechterungsprozesses, der direkt und indirekt vom Menschen verursacht wird. Regionen, die früher über große Wasserressourcen verfügten, beginnen heute, Anzeichen von Knappheit zu geben, und die Erklärung ist die Verschwendung mit übermäßiger Ausbeutung, die Verschlammung von Flüssen und die Verschmutzung der Quellen. Und all diese Probleme entstehen fast immer in der Explosion der industriellen Landwirtschaft, die dazu dient, die Millionen von Einwohnern der Städte zu ernähren. Es ist eine Tatsache, dass Urbanisierung und das grassierende Wachstum der Städte den Verbrauch und Missbrauch von Wasser deutlich erhöhen.

\section{WAS TUN ANGESICHTS DIESER VERSCHWENDUNG?}

Die Entumweltung von Wasser ist nicht nur ein technisches Problem, sondern auch eine wirtschaftliche und soziale Ordnung, da es nicht genügend Ressourcen gibt, um die flüssigen Abwasserreinigungssysteme mit der verfügbaren Technologie zu implantieren. Es ist bekannt, dass Staaten in der Lage waren, Ressourcen durch internationale Banken zu erbeuten und die ersten Schritte zugunsten unserer Quellen 
zu unternehmen, wie in Rio Grande do Sul, mit dem Staatsprogramm Pri-Guaaba und Guaéba Vive, in der Hauptstadt Porto Alegre, als Verweis auf das Problem der Wasserressourcen. Es bleibt jedoch noch viel zu tun, denn Wasser wird in allen Lebensbereichen benötigt. Süßwasserressourcen sind ein wesentlicher Bestandteil der Erdhydrosphäre und ein unverzichtbarer Bestandteil aller terrestrischen Ökosysteme und ihrer weitverbreiteten Knappheit, allmählichen Zerstörung und zunehmenden Verschmutzung der Wasserquellen in vielen Regionen der Welt. Fordern Sie Bewusstsein und Veränderungen in der Einstellung zum Wasser. Und während viele squandere und Abfall, andere bereits leiden die Folgen der Wasserknappheit. Verteilungsungleichheit und Abfall sind zwei starke Gründe, die zum Teil erklären, warum 1,4 Milliarden Menschen - fast fünfmal so viele Wiege der Bevölkerung der Vereinigten Staaten - keinen Zugang zu Trinkwasser haben und Wasser ein primärer Faktor für die Gesundheit ist. Es ist auch bekannt, dass $60 \%$ der Wasserressourcen in nur neun Ländern liegen, darunter Brasilien, aber 80 Länder, die $40 \%$ der Weltbevölkerung repräsentieren, die unter Wasserknappheit leiden. Es bedeutet jedoch nicht, dass die Vereinigten Staaten, Brasilien, Russland, Südafrika oder China, die als wasserreiche Länder gelten, nicht frei von Problemen des Wassermangels sind.

Was die Abfälle betrifft, so ist die Landwirtschaft laut Rodolfo F. Alves Pena für die weltweite Absorption von durchschnittlich $70 \%$ der Wasserversorgung verantwortlich, und dieser Index geht in den unterentwickelten Ländern auf 80 bis $90 \%$. Das Trinkwasser geht durchschnittlich $50 \%$ in den Lecks von Verteilungssystemen verloren, und wenn nichts im Sinne von Verhaltensänderungen getan wird, werden im Jahr 2025 mehr als 4 Milliarden Menschen leben, die keinen Zugang zu diesem sehr kostbaren und lebensnotwendigen Leben haben werden.

Daher ist es notwendig, die Frage der Nichtabnutzung von Wasser in der ländlichen Umgebung in einer integrierten Weise zu analysieren, in der mehrere Faktoren die Veränderung der Gewohnheit der Erzeuger beeinflussen, im Falle der ordnungsgemäßen Nutzung von Hydrinressourcen, wie Oliveira (2008) erläutert: 
Die Entscheidung und die Option für eine möglichst effiziente Nutzung der natürlichen Ressourcen im Allgemeinen und des Wassers im Besonderen sollten in integrierter Weise getroffen und mit Managementinstrumenten auf allen relevanten Ebenen in Verbindung gebracht werden. Nur so wird es möglich sein, ein Niveau zu erreichen, das eine Angemessenheit von Angebot und Nachfrage im Hinblick auf die rationelle Nutzung einer derart wertvollen Ressource ermöglicht. (OLIVEIRA, 2008, p.3)

Dieses Bewusstsein muss in den verschiedenen Bereichen der Gesellschaft stattfinden, unabhängig von der sozialen, kulturellen und intellektuellen Lage des Einzelnen. Denn heute verbraucht ein europäischer Bürger 150 Liter Wasser pro Tag, während sich ein Inder nur mit 25 Litern pro Tag begnügen muss. Ein Amerikaner verbraucht 3.000 Liter Wasser pro Tag.

Es ist gut zu betonen, dass die Verschwendung des Papiers ist mehr eine erschwerende in den gefällten Bäumen, sowie der Verbrauch von Wasser, da, um eine Tonne Frischfaser zu produzieren, ist es notwendig 44000 bis 83000 Liter Wasser. Es ist notwendig, die Verwendung von Recyclingpapier zu fördern, da für jede Tonne Papier fast eine Tonne neues Papier produziert werden kann (effizienter als das Verhältnis 2-3,5 Tonnen Bäume, um eine Tonne Jungpapier zu produzieren). Der Prozess der Umwelterziehung sollte gefördert werden.

Süßwasser, eine endliche Ressource, ist sehr anfällig und hat mehrere Verwendungsmöglichkeiten, daher muss es in integrierter Weise mit wirksamen Koordinierungs- und Umsetzungsmechanismen verwaltet werden. In den Plänen für seine Verwendung ist es notwendig, den Schutz, die Erhaltung und ein nachhaltiges und rationelles Management auf der Grundlage der Bedürfnisse und Prioritäten jeder Gemeinschaft hervorzuheben. Die Bewertung der Wasserressourcen ist die praktische Grundlage für eine integrierte und nachhaltige Bewirtschaftung sowie eine ausgewogene Untersuchung der Bedürfnisse der Bevölkerung und der Umwelt. 
Aus allen hier dargelegten Gründen ist zu bedenken, dass Wasser das wertvollste Gut dieses Jahrhunderts ist, was jedoch nicht bedeutet, dass man die "Quelle des Lebens" in ein Währungssystem verwandeln sollte, das Generator so vieler Konflikte ist, sondern vielmehr die Bemühungen zugunsten zu vereinen. bewussten Konsums und die Erhaltung dieser lebenswichtigen Ressource, um die Lebensqualität künftiger Generationen zu gewährleisten.

\section{ABSCHLIEßENDE ÜBERLEGUNGEN}

Nachhaltigkeit ist die Beziehung zwischen systemen der menschlichen Wirtschaft und der Umwelt. Die Handlungen der Menschen müssen im Einklang mit der Umwelt sein und die Erhaltung und Erhaltung gewährleisten, um die Vielfalt, Komplexität und funktionsweise des ökologischen Systems zu erhalten, das uns am Leben hält. Und nachhaltiges Denken ist notwendig, weil die natürlichen Ressourcen endlich sind. Darüber hinaus hat sich die Qualität der Ressourcen aufgrund des Bevölkerungszuwachses und des Fehlens öffentlicher Maßnahmen, die auf deren Erhaltung abzielen, immer schlechter verschlechtert. Als Beispiel wird geschätzt, dass jährlich etwa 12 Millionen Menschen aufgrund von Problemen im Zusammenhang mit der Wasserqualität sterben.

Die einzige Möglichkeit, mit der zunehmenden Verschmutzung ihrer Wasserressourcen umzuwirken, ist die Umsetzung integrierter Bewirtschaftungsstrategien, die nicht nur zur Reinigung der Wasserläufe beitragen, sondern in erster Linie auch die Verschmutzung verhindern. Bisher waren nur sehr wenige Länder in der Lage, solche Veränderungen zu praktizieren. Sie müssen wachsam sein. Wir müssen Verschwendung vermeiden, umweltschädliche Prozesse stören und neue Möglichkeiten der Kontrolle, Erfassung und Verteilung schaffen.

Es bedarf einer kulturellen und ethischen Antwort. Die Situation, durch die die Welt vorbeigeht, ist nichts anderes als ein ausgeklügelterlebensstil, in dem das, was man haben will, "muss haben" und damit die Beschleunigung von Fabriken und Industrien verursacht, die zu den größten Wasserverbrauchern gehören, die nur an die Landwirtschaft und den Polyesterverbrauch verlieren. plastik. Im TWENTIETH- 
Jahrhundert wuchs die Bevölkerung dreimal, während der Wasserverbrauch um sechs stieg. Selbst wir Brasilianer, die $16 \%$ des gesamten Wassers der Welt haben, leiden unter Rationierungen aufgrund schlechter Ressourcenverwaltung und unzureichender Infrastruktur. Die trockenen Länder stehen vor beängstigenderen Herausforderungen wie dem Nahen Osten, wo es Gebiete gibt, in denen Knappheit das sozioökonomische Wachstum einschränkt.

Gemeinsame Bewirtschaftungsmaßnahmen für die Nutzung von Land und Wasserressourcen sollten häufiger sein, um die Effizienz der Nutzung von Bewässerungswasser zu erhöhen und so Bodenerosion, Überschwemmungen, Sedimentation, Versalzung und die schädlichen Auswirkungen von Pestizide und Düngemittel in Organismen. Darüber hinaus hat die Umweltzerstörung zu einer sich abzeichnenden Umweltpathologie geführt, und Krankheiten wie Cholera und Dengue sind durch die Kontamination von Luft, Wasser und Böden sowie durch den Einsatz giftiger und gefährlicher Stoffe wie Cholera und Dengue stärker verursacht worden. Pestizide und andere spezielle Pflegeprodukte. Wie wir bisher gesehen haben, ist die Degradierung nicht nur die Umwelt, sondern auch der Mensch, dessen Werte verloren gehen, wo die Notwendigkeiten nicht mehr das Grundlegende sind, sondern das Überflüssige, wo Unrecht das Richtige ist. Hier werden wir die Umwelterziehung als Priorität betrachten, um die Ziele der nachhaltigen Entwicklung zu erreichen. Die Konferenz von Tiflis, die sich 1977 mit Umwelterziehung auf internationaler Ebene befasst, macht die EA zur Bildung eines Bewusstseins, das auf einer neuen Ethik beruht, die jeder Ausbeutung widerstehen wird. Das ist es, was die Menschheit braucht, um ihre Handlungen, ihre Investitionen, ihre Haltungen, ihre Werte zu ändern, um zu erkennen, dass sie nicht nur an sich selbst denkt, dass sie in der Lage sein wird, ihren Generationen eine gesunde Zukunft zu geben.

Wir wissen, dass der Mann der Hauptursache für so viel Ungleichgewicht ist. Der Mann störte so völlig die natürliche Umgebung, in der er sich bewegte, dass er seinen Platz in diesem System nicht mehr kennt, außer natürlich als Störungsfaktor. Bisher bestanden unsere Versuche, das Medium zu beherrschen, aus einfachen Landschaftsreformen, die ungeschickt und unreflektiert durchgeführt wurden; 
Während andere menschliche Aktivitäten schädliche, unvorhergesehene und schlecht verstandene Nebenwirkungen produziert haben. Alle Ozeane wurden von Menschen, der Atmosphäre und sogar den abgelegenen Gletscherschichten verschmutzt. Die meisten Flüsse sind mehr oder weniger verschmutzt und viele von ihnen, wie der TietéFluss und der Tiber, wurden in Freiluftabwässer umgewandelt. Barbie Nadeau, Journalistin von Newsweek im Jahr 2002 war in Rom und gibt ihre Aussage über den Tiber "... Sie schwimmen tote Fische dezimiert, nicht bekannt für was. Der Gestank ist überall, ein öliger Rückstand lässt die Aale und die toten Karpfen leuchten "und schließt:"... Wenn ein Fluss die symbolische Linie des Lebens einer Stadt ist, dann ist Rom in einer ziemlich schlechten Situation. " Der Fall ist nicht, das Medium zu dominieren, sondern zu wissen, ob die Natur mit einem bestimmten Anschein von Ordnung bewahrt werden kann und ob die Zivilisation in der Lage ist, die Transformation zu überleben, die in der Natur provoziert. Die kulturelle Evolution hat uns allmählich zu der Anerkennung veranlasst, dass die Humanisierung des Planeten nur gelingen kann, wenn grundlegende ökologische Gesetze respektiert werden.

Es ist an der Zeit, über sich ändernde Gewohnheiten nachzudenken. Das Gefühl, dass wir eine einzige Kette bilden; Wenn Sie ein Glied brechen, hat die Kette keinen Sinn mehr. Jeder braucht jeden. Die indischen Gelehrten, vor Tausenden von Jahren, sagten bereits, dass das Ganze in den Teilen enthalten ist, und so sehr die Dinge diversifiziert und plural erscheinen, in der Tat, sie sind Aspekte der gleichen und einzigen Realität. Hier ist ein Satz, der von uns allen vermittelt werden muss, damit wir die Dynamik des Lebens verstehen und sehen können, dass es kein Ich und ja, die Knoten gibt. Bisher wurde die Natur kostenlos genutzt, und dank ihr produzieren und leben wir. Im Panorama des Wachstums der Weltbevölkerung hat sie jedoch ihren gebührenden Wert nicht erkannt.

In Bezug auf die Bewässerung, verantwortlich für die Abfälle und konsequente Verpflichtung der Federn, um die Auswirkungen seiner unzureichenden Nutzung zu minimieren, betont Rebouas (2001), dass es notwendig ist, eine effizientere und rationelle Planung der Verwendung von Bewässerung und Optimierung der verwendeten Geräte. Es kann auch die Stromkosten senken. 
Eine weitere neuere Praxis in Brasilien, die an Stärke gewonnen hat, ist die Verwendung von Wiederverwendungswasser, um den Bedarf an Bewässerung verschiedener Nahrungspflanzen zu decken. Hespanhol (2002) ergänzt, dass in mehreren Ländern, in denen diese Technik zum Einsatz kommt, die Produktivität bei gut geplanter Planung deutlich steigt.

Jede Form der effizienten Nutzung von Wasser zur Minimierung von Abfällen im Feld hängt direkt von der Planung des Erzeugers ab. Die Planung ist unerläßlich, um die verschiedenen Wassernutzungen zu harmonisieren, die verschiedenen Produktionssektoren zu ermöglichen, die Menge und Qualität der hydrischen Ressourcen zu überwachen und die Gesamteffizienz der Nutzung zu verbessern (PAZ et al., 2000).

Zweite (FURRIELA,2001) Die erste große Herausforderung, die sich nicht nur für den ländlichen Produzenten, sondern auch für die gesamte Menschheit stellt, ist das Bewusstsein, dass es keine unerschöpfliche Trinkwasserversorgung auf dem Planeten gibt. Einige Maßnahmen sollten gefördert werden, um die Versorgung mit Wasser in Mengen und akzeptablen Standards zu gewährleisten. Die große Herausforderung von heute besteht darin, den Grad der Wasserdegradation durch Maßnahmen wie Erhaltung, Verbesserung der grundlegenden sanitären Einrichtungen, Reduzierung des Pestizideinsatzes, sauberere industrielle Produktion und Verbrauchsmanagement zu verringern.

Jeder Tag wird deutlicher die Notwendigkeit, Wasser als ein wirtschaftliches Gut zu betrachten, in dem seine Verwaltung auf Denprinzipien der wirtschaftlichen Effizienz basieren sollte, in einer Weise, die die Nachfrage aus einer Perspektive der Nachhaltigkeit erfüllt.

Außerdem sei es notwendig, eine neue Denkweise und ein neues Handeln zu etablieren, wobei unterschiedliche Gewohnheiten, Verwendungen und Bräuche angenommen würden, wobei das allgemeine Ziel das Wirtschaftswachstum sei, das auf dem nachhaltigen Wasserverbrauch beruhe, und so den Schutz der Die noch erhaltenen Quellen und die Wiederherstellung der bereits Geschädigten, weist er 
darauf hin, dass dies nur nicht ausreicht, und es ist notwendig, viel mehr zu tun, um dieses Ziel zu erreichen, daher sollte Wasser als eine soziale und ökologische Priorität behandelt werden, da es notwendig ist, Die Versorgung der brasilianischen Wasserressourcen, da der steigende Bedarf an Weltwasser dem Land die Möglichkeit geben wird, sein Wachstum und seine Entwicklung zu nutzen und einen wesentlichen Beitrag zum Leben und zu jeder produktiven Tätigkeit zu leisten.

\section{BIBLIOGRAPHISCHE HINWEISE}

AGENDA 21. The Earth Summit Strategy to save our Planet. Ed. Daniel Sitarz, Earthpress, 321p. Boulder Colorado, 1994.

AGÊNCIA NACIONAL DAS ÁGUAS. Disponível em: <www.ana.gov.br> Acesso em: $20 / 07 / 2019$

Baía de todas as águas - Preservação e Gerenciamento Ambiental na Bacia Hidrográfica do Guaíba - Pró-Guaíba, RS/1998.

EBC. Disponível em: <www.ebc.com.br/.../mais-de-75-das-florestas-primarias-domundo-nao-existem-mais >Acesso em:19/07/2019

FURRIELA, R. B. EDUCAÇÃO PARA O CONSUMO SUSTENTÁVEL. Ciclo de Palestras sobre Meio Ambiente - Programa Conheça a Educação do Cibec/InepMEC/SEF/COEA, 2001.

HESPANHOL, I. Potencial de Reuso da Água No Brasil, Agricultura, Industria, Municípios, Recarga de Aquíferos. RBRH- Revista Brasileira Dos Recursos Hídricos. Vol. 7 n. 4, 2002

JUSBRASIL. Disponível em: <https://www.jusbrasil.com.br/topicos/10603350/artigo90-do-decreto-n-24643-de-10-de-julho-de-1934> Acesso em 20/07/2019 
MUNDO

DA

EDUCAÇÃO.

Disponível

em:

$<$ https://mundoeducacao.bol.uol.com.br/geografia/consumo-agua-no-mundo.htm> Acesso em 12 de setembro de 2018.

OLIVEIRA, E. C. A Gestão Dos Recursos Hídricos Sob a Perspectiva da Descentralização. ANAP BRASIL - REVISTA CIENTÍFICA, ano 1 nº 1, 2008.

PORTAL FMU. Disponível em: <https://portal.fmu.br/reciclagem-no-brasil-panoramaatual-e-desafios-para-o-futuro/> Acesso em: 20/07/2019

PUC RS. Disponível em: $<$ www.pucrs.br/edipucrs/online/planetaagua/planetaagua.pdf> Acesso em: $20 / 07 / 2019$

REVISTA EDUCAÇÃO AMBIENTAL EM AÇÃO. Disponível em: <http://www.revistaea.org/artigo.php?idartigo=1271> Acesso em: 17/07/2019

REBOUÇAS, A. C. Água e desenvolvimento rural. ESTUDOS AVANÇADOS, 2001.

REBOUÇAS, A.C. Água no Brasil: Abundância, Desperdício e Escassez.BAHIASalvador, v. 13, n. ESPECIAL, p. 341-345, 2003 ANÁLISE \& DADOS

SILVA,J. S. Água e Agricultura Irrigada. Palestra-Seminário Agricultura Irrigada Com Desenvolvimento Sustentável-Universidade Federal De Uberaba-Em 24-09-2009. Disponível em: http:www.irrigacao.org.brdocdownloadAgua\%20Agricultura\%20Irrigada_JoseSilverio. pdf.. Acessado em: 20/07/2019

TUCCI, C. E. M. Gestão da água no Brasil. Brasília: UNESCO, 2001. 156p.(1. Hidrologia - Brasil II. Hespanhol, Ivanildo III. Cordeiro Netto,

VICTORINO, C. J. A. Planeta água morrendo de sede: uma visão analítica na metodologia do uso e abuso dos recursos hídricos. Porto Alegre: EDIPUCRS, 2007.231 p. (1. Água - Uso. 2. Água - Qualidade. 3. Recursos). 
Eingereicht: Juni 2019.

Genehmigt: August 2019. 